\title{
Comprehensive analysis of microRNA-mRNA co-expression in circadian rhythm
}

\author{
Young-Ji ${ }^{1,2 *}$, Jung Hwan Sung ${ }^{3 *}$, Suk Chan Lee ${ }^{4}$, \\ Young-Ju Lee ${ }^{5}$, Yeun Joo Choi $^{5}$, \\ Woong-Yang Park ${ }^{5,6}$, Hee Sup Shin ${ }^{4}$ \\ and Ju Han Kim ${ }^{1,5,7}$ \\ ${ }^{1}$ Seoul National University Biomedical Informatics (SNUBI) \\ Seoul National University College of Medicine \\ Seoul 110-799, Korea \\ ${ }^{2}$ Interdisciplinary Program in Bioinformatics \\ Seoul National University \\ Seoul 151-742, Korea \\ ${ }^{3}$ Program in Biological and Biomedical Sciences \\ Harvard Medical School \\ Boston, MA 02115, USA \\ ${ }^{4}$ National Creative Research Initiative Center for Calcium and Learning \\ Korea Institutes of Science and Technology \\ Seoul 136-791, Korea \\ ${ }^{5}$ Human Genome Research Institute \\ ${ }^{6}$ Department of Biochemistry and Molecular Biology \\ Seoul National University College of Medicine \\ Seoul 110-799, Korea \\ ${ }^{7}$ Corresponding author: Tel, 82-2-740-8320; \\ Fax, 82-2-740-5947; E-mail, juhan@ @nu.ac.kr \\ *These authors contributed equally to this work. \\ DOI 10.3858/emm.2009.41.9.070
}

Accepted 23 April 2009

Abbreviations: Cry, cryptochrome; GEO, gene Expression Omnibus; miRNA, microRNA; Per, period

\begin{abstract}
To investigate the potential role of microRNA (miRNA) in the regulation of circadian rhythm, we performed microarray-based expression profiling study of both miRNA and mRNA in mouse liver for $\mathbf{4 8} \mathrm{h}$ at 4-hour intervals. Circadian miRNA-mRNA target pair is defined as the pair both elements of which show circadian expression patterns and the sequence-based target relationship of which can be predicted. Circadian initiators, Clock and Bmal1, showed inversely correlated circadian expression patterns against their corresponding miRNAs, miR-181d and miR-191, targeting them. In contrast, circadian suppressors, Per, Cry, CKle and Rev-erba, exhibited positively correlated circadian expression patterns to their corresponding
\end{abstract}

miRNAs. Genomic location analysis revealed that intronic region showed higher abundance of cyclic than non-cyclic miRNAs targeting circadian genes while other (i.e., 3'-UTR, exon and intergenic) regions showed no difference. It is suggested that miRNAs are involved in the regulation of peripheral circadian rhythm in mouse liver by modulating Clock:Bmal1 complex. Identifying specific miRNAs and their targets that are critically involved in circadian rhythm will provide a better understanding of the regulation of circadian- clock system.

Keywords: circadian rhythm; gene expression profiling; gene expression regulation; liver; mice; microRNA

\section{Introduction}

The circadian system in higher organisms temporally orchestrates rhythmic changes in a vast number of genes and gene products in different organs (Reppert and Weaver, 2001). Circadian rhythms of gene expression occur widely in peripheral organs. Analysis of "circadian proteome" revealed that the portion of cycling proteins is rather higher than that found in "circadian transcriptome", and that many of the cycling proteins show a constant abundance at the level of the mature transcript (Reddy et al., 2006). This discrepancy indicates that posttranscriptional and posttranslational mechanisms are important components of circadian rhythmicity. Therefore, it is important to identify the molecular basis of miRNAmediated gene regulation of circadian timing in peripheral tissues.

MicroRNAs (miRNAs) are small, non-coding RNAs that act as potent silencers of gene expression (Lee et al., 1993; Pasquinelli et al., 2000; Lagos-Quintana et al., 2001; Lau et al., 2001; Lee and Ambros, 2001; Bartel, 2004). They recognize target mRNAs by base-pairing and thereby regulate their expression (Lewis et al., 2003; Kiriakidou et al., 2004). It is estimated that up to $30 \%$ of protein-coding genes are regulated by miRNAs (Krek et al., 2005; Lewis et al., 2005). They are involved in various biological processes such as developmental timing and patterning, apoptosis, cell proliferation, organ development and tumorigenesis (Hornstein et al., 2005; Wienholds and 
Plasterk, 2005). Most miRNAs are expressed in a tissue-dependent manner. With microarrays those tissue-specific miRNAs can be identified (Thomson et al., 2004).

The breadth of genetic regulatory effects potentially mediated by miRNAs and their central role in diverse cellular processes have led to the hypothesis that gene-expression changes of specific miRNAs may be correlated with the regulation of the circadian-clock initiators that are critically involved in circadian rhythm. The aim of the present study is to identify and characterize the miRNAs involved in the regulation of circadian rhythm. Not all of miRNAs showing circadian patterns are involved in circadian rhythm and miRNAs showing non-circadian patterns may also be involved in circadian rhythm. However, characterizing and analyzing such miRNAs is not considered in this paper. Here, we focus entirely on miRNAs that simultaneously have circadian expression patterns and target circadian genes. We asked the following questions: Can we find miRNAs having diurnal expression pattern? How can we characterize the cyclic miRNAs in terms of target relationships and/or genomic locations? Is miRNA a regulator of circadian genes?

\section{Results}

\section{MicroRNAs showing cyclic expression patterns and their targets}

We found $85(13.3 \%)$ among the 640 miRNAs probed having circadian expression patterns by the two statistical methods $(r>0.7$ and $P$ value $<$ 0.05 , see Method) (Table 1 ). Table 1 shows 70 miRNAs having circadian expression patterns

Table 1. Micro-RNAs with cyclic expression patterns and their genomic locations and target genes.

\begin{tabular}{|c|c|c|c|c|c|}
\hline \multirow{2}{*}{$\begin{array}{l}\text { Peak hour } \\
\text { (day-1/day-2) }\end{array}$} & \multirow{2}{*}{ miRNA } & \multirow{2}{*}{$\begin{array}{l}\text { Genomic } \\
\text { location }\end{array}$} & \multirow{2}{*}{$\begin{array}{c}\text { Verified targets } \\
\text { TarBase }\end{array}$} & \multicolumn{2}{|c|}{ Predicted targets } \\
\hline & & & & MiRanda & TargetScan \\
\hline $4 / 28$ & hsa-miR-129 & G & & & \\
\hline \multirow[t]{2}{*}{$(n=4)$} & rno-miR-450 & 1 & & Bmal1 & \\
\hline & rno-miR-664 & I/G & & & \\
\hline \multirow{29}{*}{$\begin{array}{l}8 / 32 \\
(n=40)\end{array}$} & hsa-let-7i & $\mathrm{G}$ & KRAS & & \\
\hline & hsa-miR-142-5p & G & & & \\
\hline & hsa-miR-146b & $\mathrm{G}$ & & Per3, Cry1, Timeless & Timeless \\
\hline & hsa-miR-150 & 3'-UTR & & & \\
\hline & hsa-miR-181d & G & & Clock, Timeless & Per2, Timeless \\
\hline & hsa-miR-200b & G & & Clock, Timeless & Timeless \\
\hline & hsa-miR-208 & I & & Npas2 & \\
\hline & hsa-miR-20b & G & $E 2 F 1$ & & \\
\hline & hsa-miR-28 & I & & Timeless & Timeless \\
\hline & hsa-miR-324-3p & I & & Rev-erba, Npas2 & \\
\hline & hsa-miR-339 & 1 & & & \\
\hline & hsa-miR-365 & I/G & & Clock & \\
\hline & hsa-miR-483 & I & & Clock, Timeless, Npas2 & Timeless \\
\hline & hsa-miR-491 & I & & & Rev-erba \\
\hline & hsa-miR-500 & I & & & \\
\hline & hsa-miR-520g & G & & Npas2 & \\
\hline & hsa-miR-542-3p & G & & & \\
\hline & hsa-miR-92 & I/G/3'-UTR & & & \\
\hline & hsa-miR-99b & $\mathrm{G}$ & & & \\
\hline & mmu-miR-17-3p & G & & Cry2, CKle & \\
\hline & mmu-miR-187 & G & & & \\
\hline & mmu-miR-20b & G & & & \\
\hline & mmu-miR-345 & $G$ & & & \\
\hline & mmu-miR-383 & $\mathrm{G}$ & & Cry1 & \\
\hline & mmu-mir-429 & I/E & & & \\
\hline & mmu-miR-431 & 3'-UTR & Rtl1/Peg11 & & \\
\hline & mmu-miR-483 & I & & Cry1 & \\
\hline & mmu-miR-503 & G & & & \\
\hline & mmu-miR-99a & G & & & \\
\hline
\end{tabular}


Table 1. Continued.

\begin{tabular}{|c|c|c|c|c|c|}
\hline \multirow{2}{*}{$\begin{array}{l}\text { Peak hour } \\
\text { (day-1/day-2) }\end{array}$} & \multirow{2}{*}{ miRNA } & \multirow{2}{*}{$\begin{array}{l}\text { Genomic } \\
\text { location }\end{array}$} & \multirow{2}{*}{$\begin{array}{c}\text { Verified targets } \\
\text { TarBase }\end{array}$} & \multicolumn{2}{|c|}{ Predicted targets } \\
\hline & & & & MiRanda & TargetScan \\
\hline & rno-miR-151 & 1 & & & \\
\hline & rno-miR-20b & $\mathrm{G}$ & & & \\
\hline & rno-miR-333 & I & & Per1 & \\
\hline & rno-miR-352 & G & & & \\
\hline & rno-miR-376b & $\mathrm{G}$ & & & \\
\hline $12 / 36$ & hsa-miR-136 & G & Rt/1/Peg11 & & \\
\hline \multirow[t]{11}{*}{$(n=13)$} & hsa-miR-181a & I & & Clock, Timeless & Per2, Timeless \\
\hline & hsa-miR-191 & I & & Bmal1 & \\
\hline & hsa-miR-26b & I & SMAD1 & & \\
\hline & hsa-miR-302b-AS & I & & & \\
\hline & hsa-miR-328 & I & & Per1 & \\
\hline & hsa-miR-422b & I & & & \\
\hline & hsa-miR-7 & 1 & & & \\
\hline & mmu-miR-140 & I & & & \\
\hline & mmu-miR-140-AS & I & & & \\
\hline & mmu-miR-221 & $\mathrm{G}$ & $K I T$ & & \\
\hline & mmu-miR-350 & I & & Timeless & \\
\hline \multirow{7}{*}{$\begin{array}{l}16 / 40 \\
(n=9)\end{array}$} & hsa-miR-103 & I & $F B X W 1 B$ & & \\
\hline & hsa-miR-105 & I & & & Timeless \\
\hline & hsa-miR-135b & 1 & & Bmal1 & \\
\hline & hsa-miR-182 & $\mathrm{G}$ & & Clock & CLOCK, CKIe \\
\hline & hsa-miR-362 & I & & & \\
\hline & hsa-miR-432-AS & $G$ & & & Rev-erba \\
\hline & mmu-miR-467 & I & & & \\
\hline \multirow{13}{*}{$\begin{array}{l}20 / 44 \\
(n=18)\end{array}$} & hsa-miR-149 & I & & & \\
\hline & hsa-miR-212 & G & & & \\
\hline & hsa-miR-376b & G & & Bmal1 & \\
\hline & hsa-miR-501 & I & & & \\
\hline & mmu-miR-202 & $\mathrm{G}$ & & Bmal1 & \\
\hline & mmu-miR-211 & I & & & \\
\hline & mmu-miR-292-3p & $G$ & & Npas2 & \\
\hline & mmu-miR-376b-AS & $G$ & & & \\
\hline & mmu-miR-463 & G & & & \\
\hline & mmu-miR-471 & G & & Per3 & \\
\hline & rno-miR-344 & $\mathrm{G}$ & & & \\
\hline & rno-miR-377 & $G$ & & & \\
\hline & rno-miR-501 & $\mathrm{G}$ & & & \\
\hline $\begin{array}{l}24 / 48 \\
(n=1)\end{array}$ & hsa-miR-302a-AS & I & & & \\
\hline
\end{tabular}

Omitted are the 15 miRNAs without valid probe information including ambi-miR-7055 (4/28 h), ambi-miR-10133, ambi-miR-11541, ambi-miR-11835, ambi-miR-13100, ambi-miR-13258, ambi-miR-13268 (8/32 h), ambi-miR-7083 (12/36 h), ambi-miR-11786, ambi-miR-12152 (16/40 h) and ambi-miR-408, ambi-miR-7067, ambi-miR-7068-1, ambi-miR-7086, ambi-miR-9873 (20/44 h). G indicates intergenic, I intronic and E exonic regions.

including 39 human and 10 rat probes as well as 21 mouse probes. The 10 rat probes do not have homologue in mouse. Most of the human probes (38/39), however, were human homologues of mouse miRNA, as explained in the method section. Some of the one human and 10 rat probes without having mouse homology may be 'false positives'. Others may be 'true positive' mouse miRNAs having human or rat homologues that have not yet been determined. It may be worth validating as new mouse miRNA genes were discovered by homology search (Weber, 2005).

In the six four-hour interval groups ranging from ZT0 (the beginning of the subjective day) to ZT24 (the end of the subjective night, equal to ZTO), we found the following distribution of peaks (Figure 1): four genes in ZT4, 40 in ZT8, 13 in ZT12, nine in ZT16, 18 in ZT20 and one in ZT24. 

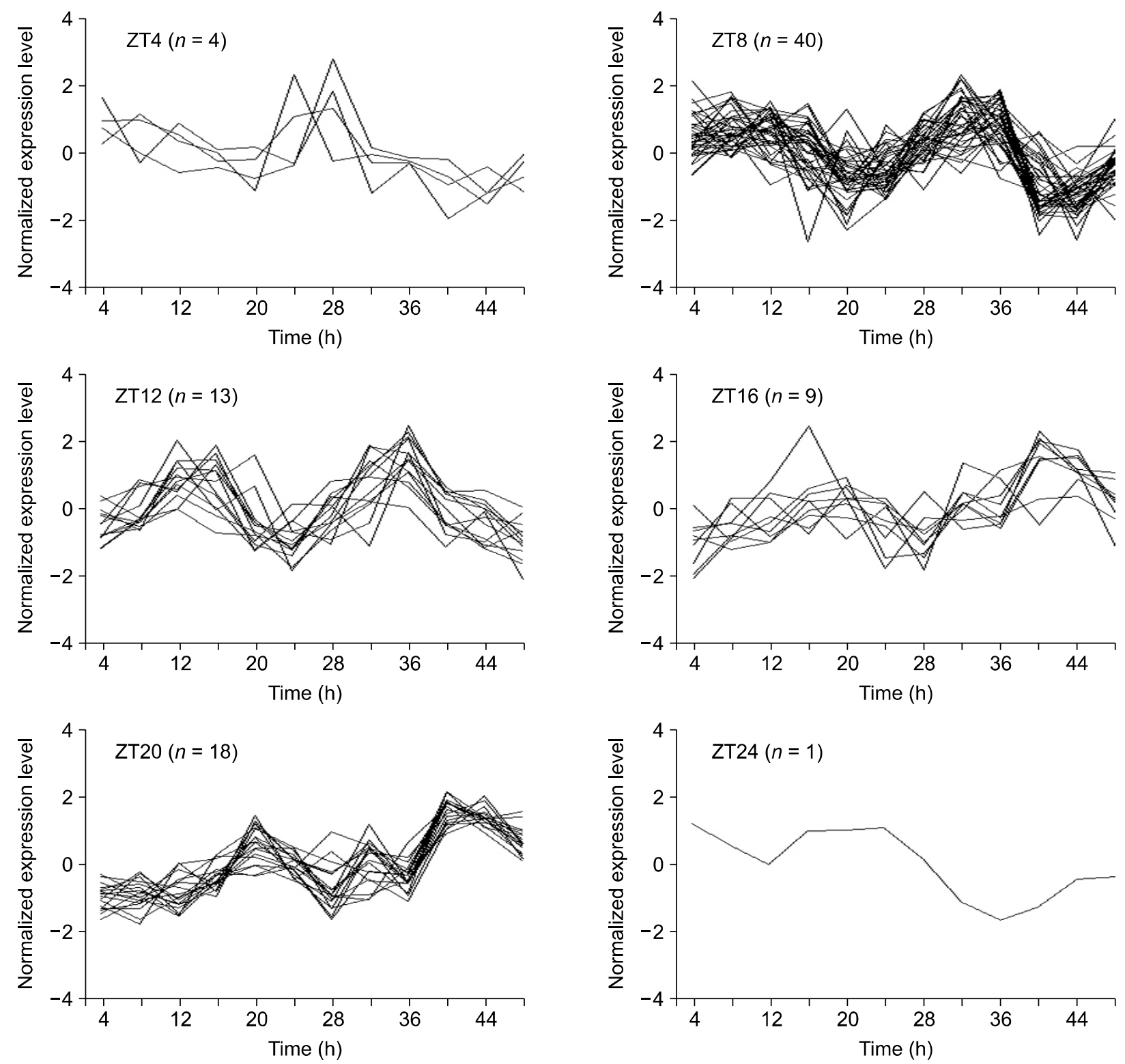

Figure 1. miRNAs showing circadian expression patterns in mouse liver. Cosinor analysis and correlation analysis determined 85 out of 640 miRNAs showing circadian expression patterns. MiRNAs are binned into six phases. The data in this plot were transformed so that it had a zero mean and a standard deviation of 1. Horizontal axis represents hours passed after the first subjective dawn.

Using TarBase (Sethupathy et al., 2006), we found that seven among the 85 cyclic miRNAs have experimentally validated mRNA targets. Table 1 demonstrates that they are oncogenes like KRAS and $K I T$ and cell cycle regulatory protein like E2F1. $F B X W 1 B$ belonging to $\mathrm{F}$-box protein family is one of the targets. FBXL3 in the same F-box protein family has been reported to control the oscillation of the circadian clock by directing the degradation of cryptochrome proteins (Siepka et al., 2007). This finding suggests that cyclic miRNAs may be asso- ciated with circadian rhythm, cell cycle and oncogenes.

\section{MicroRNAs targeting core circadian clock genes}

Core circadian clock genes are defined as the genes whose protein products are necessary components for the generation and regulation of circadian rhythm (Lowrey and Takahashi, 2004). We selected twelve circadian genes from the current list of mammalian clock genes, Clock, Bmal1, Per1, 
Per2, Per3, Cry1, Cry2, Rev-erba, CKle, Timeless, Npas2 and Bmal2 (Lowrey and Takahashi, 2004). To find miRNAs targeting these core clock genes, we used MiRanda (John et al., 2004) based on target prediction. We found that 205 (32\%) among the 640 miRNAs probed are potentially targeting them.

We defined circadian miRNA-mRNA target pair as the pair both elements of which show circadian expression patterns $(r>0.7$ and $P$ value $<0.05$, see Method) and whose sequence-based target relationship is established. Target relationships between the cyclic miRNAs and mRNAs are established by using MiRanda (John et al., 2004). Table 1 shows that 33 significant miRNA-mRNA target pairs are established between 24 miRNAs and 10 core clock genes. Per2 and Bma/2 among the core circadian clock genes are excluded because no cyclic miRNA targets them. When we applied TargetScan (Lewis et al., 2003) instead of MiRanda (John et al., 2004), 13 pairs are detected. MiRanda (John et al., 2004) and TargetScan (Lewis et al., 2003) require different base-pairing rules, crossspecies conservation to identify potential miRNA binding sties. In order to compensate for limitations of their computational target prediction programs, we simultaneously showed the results by both of them.

Table 2 summarizes the 33 pairs with positive ( $n$ $=15)$ and negative $(n=18)$ correlation of expression between cyclic miRNAs and target core clock genes. Eleven among the 15 'negatively' correla-

Table 2. Correlation of expression pattern between cyclic miRNA and target circadian gene.

\begin{tabular}{|c|c|c|c|c|c|}
\hline miRNA & Peak hour (day-1/day-2) & ${ }^{\mathrm{a}}$ Genomic location & Predicted targets & Pearson's $r$ & $P$ value \\
\hline hsa-miR-181d & $8 / 32$ & I & Clock $^{\ddagger}$ & -0.82 & 0.001 \\
\hline hsa-miR-191 & $12 / 36$ & I & $B m a l 1^{\dagger}$ & -0.68 & 0.015 \\
\hline hsa-miR-181a & $12 / 36$ & 1 & Clock & -0.66 & 0.020 \\
\hline hsa-miR-365 & $8 / 32$ & $\mathrm{I} / \mathrm{G}$ & Clock & -0.51 & 0.087 \\
\hline hsa-miR-483 & $8 / 32$ & 1 & Clock & -0.51 & 0.092 \\
\hline hsa-miR-200b & $8 / 32$ & I & Clock & -0.42 & 0.170 \\
\hline hsa-miR-182 & $16 / 40$ & G & Clock & -0.34 & 0.273 \\
\hline hsa-miR-181d & $8 / 32$ & 1 & Timeless & -0.25 & 0.438 \\
\hline hsa-miR-483 & $8 / 32$ & I & Npas2 & -0.20 & 0.531 \\
\hline hsa-miR-520g & $8 / 32$ & I & Npas2 & -0.17 & 0.605 \\
\hline mmu-miR-350 & $12 / 36$ & I & Timeless & -0.14 & 0.658 \\
\hline hsa-miR-324-3p & $8 / 32$ & i & Npas2 & -0.14 & 0.673 \\
\hline hsa-miR-208 & $8 / 32$ & I & Npas2 & -0.11 & 0.739 \\
\hline hsa-miR-181a & $12 / 36$ & I & Timeless & -0.06 & 0.850 \\
\hline hsa-miR-146b & $8 / 32$ & $\mathrm{G}$ & Timeless & -0.05 & 0.878 \\
\hline hsa-miR-324-3p & $8 / 32$ & I & Rev-erba** & 0.80 & 0.002 \\
\hline mmu-miR-383 & $8 / 32$ & $G$ & Cry $1^{* *}$ & 0.75 & 0.005 \\
\hline hsa-miR-328 & $12 / 36$ & I & Per1 ${ }^{* *}$ & 0.73 & 0.008 \\
\hline hsa-miR-146b & $8 / 32$ & $G$ & Per3 ${ }^{\star *}$ & 0.72 & 0.008 \\
\hline mmu-miR-17-3p & $8 / 32$ & 1 & $C K l e^{*}$ & 0.69 & 0.014 \\
\hline hsa-miR-146b & $8 / 32$ & $\mathrm{G}$ & Cry $1^{*}$ & 0.65 & 0.021 \\
\hline mmu-miR-202 & $20 / 44$ & $\mathrm{G}$ & Bmal1 & 0.48 & 0.116 \\
\hline mmu-miR-17-3p & $8 / 32$ & $G$ & Cry2 & 0.40 & 0.203 \\
\hline mmu-miR-483 & $8 / 32$ & I & Cry 1 & 0.38 & 0.219 \\
\hline rno-miR-333 & $8 / 32$ & I & Per1 & 0.29 & 0.356 \\
\hline mmu-miR-292-3p & $20 / 44$ & G & Npas2 & 0.29 & 0.369 \\
\hline hsa-miR-200b & $8 / 32$ & I & Timeless & 0.24 & 0.450 \\
\hline hsa-miR-483 & $8 / 32$ & i & Timeless & 0.17 & 0.600 \\
\hline hsa-miR-376b & $20 / 44$ & $\mathrm{G}$ & Bmal1 & 0.16 & 0.614 \\
\hline hsa-miR-135b & $16 / 40$ & 1 & Bmal1 & 0.15 & 0.641 \\
\hline hsa-miR-28 & $8 / 32$ & I & Timeless & 0.11 & 0.738 \\
\hline rno-miR-450 & $4 / 28$ & 1 & Bmal1 & 0.08 & 0.800 \\
\hline mmu-miR-471 & $20 / 44$ & $\mathrm{G}$ & Per3 & 0.00 & 0.993 \\
\hline
\end{tabular}

Per2 and Bmal2 among the core circadian clock genes are excluded from this table because no cyclic miRNA targets them. Table 2 is sorted in an ascending order of $P$ values separately for those have positive and negative Pearson correlation coefficients. ${ }^{a} \mathrm{G}$ indicates intergenic and $\mathrm{I}$ intronic regions. ${ }^{*} P<$ 0.05 and ${ }^{* *} P<0.01$ with positive Pearson correlation coefficient by cosinor analysis. ${ }^{\dagger} P<0.05$ and ${ }^{\ddagger} P<0.01$ with negative Pearson correlation coefficient by cosinor analysis. 
ted pairs $(73.3 \%)$ targeted Clock, Bmal1 and Npas2, functioning as circadian initiators. The rest four pairs targeted Timeless. Timeless appeared four times in negatively and three times in positively correlated pairs in total but with very poor statistical significance $(P$ value $>0.4)$. In contrast, 13 among the 18 'positively' correlated pairs ( $72.2 \%)$ targeted circadian suppressors like Per1, Per3, Cry1, Cry2, CKle, Rev-erba and Timeless. The rest five pairs targeted Npas2 and Bmal1 but with poor statistical significance $(P$ value $>0.3)$. In summary, among the nine pairs with $P$ values smaller
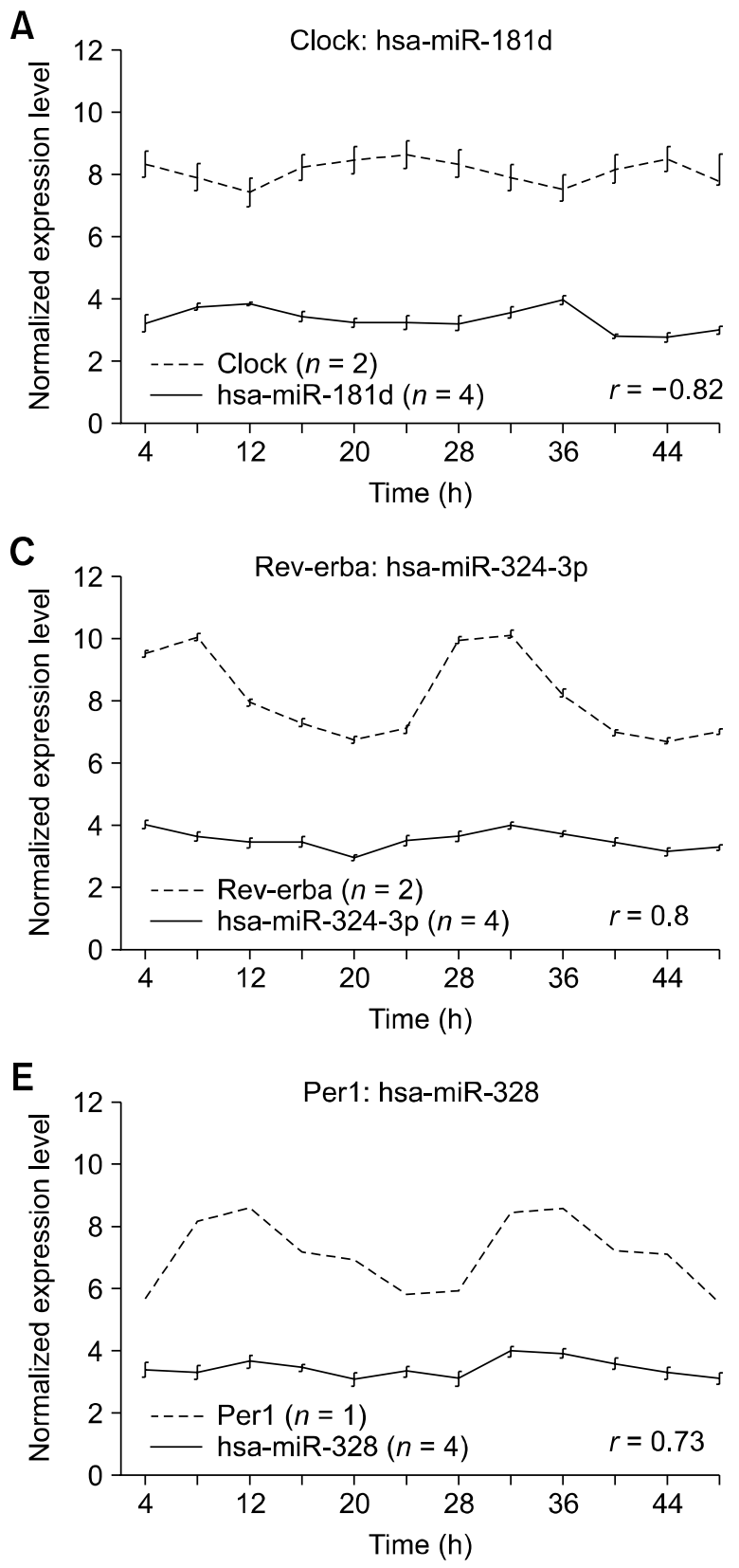

than 0.05 , statistically significant negative correlation was found only in circadian initiators, Clock $(r$ $=-0.82, P$ value $=0.001 ; r=-0.66, P$ value $=0.020)$ and Bmal1 $(r=-0.68, P$ value $=0.015)$. Statistically significant positive correlation was found only in circadian suppressors, Per3 $(r=0.72, P$ value $=$ $0.008)$, Cry1 $(r=0.65, P$ value $=0.021)$, Rev-erba $(r=0.80, P$ value $=0.002)$, CKle $(r=0.69, P$ value $=0.014)$ and Per1 $(r=0.73, P$ value $=0.008)($ Table 2). When we increased the $P$ value threshold to 0.10 , two 'negatively' correlated pairs targeting Clock $(r=-0.51, P$ value $=0.087)$ were correctly
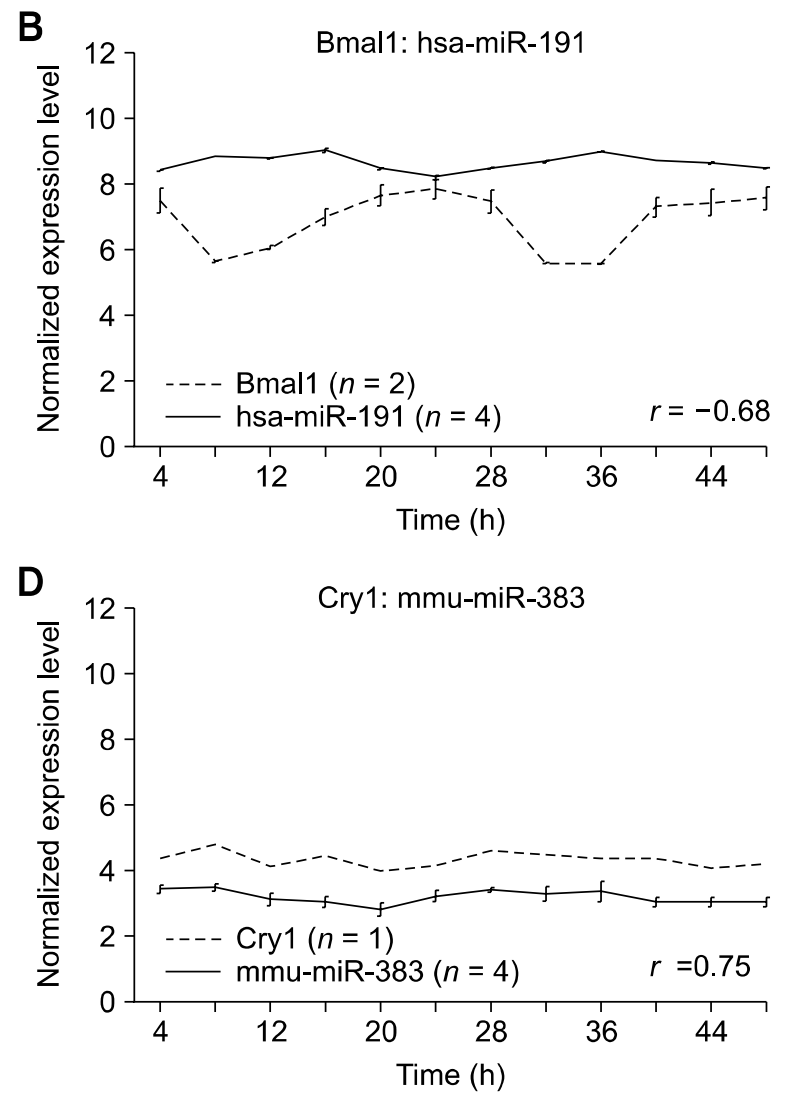

Figure 2. Expression profile of cyclic miRNAs and the target circadian genes. Expression profiles of the miRNAs targeting Clock and Bmal1, which functions as the circadian transcription factors, tend to show significant and inverse correlations with those of the target circadian genes $(P$ value $<0.01$ ). In contrast, the components of the circadian negative feedback loop including Rev-erba, Cry1, and Per1 showed significant positive correlation in expression patterns with the miRNAs targeting them ( $P$ value $<0.01)$. 

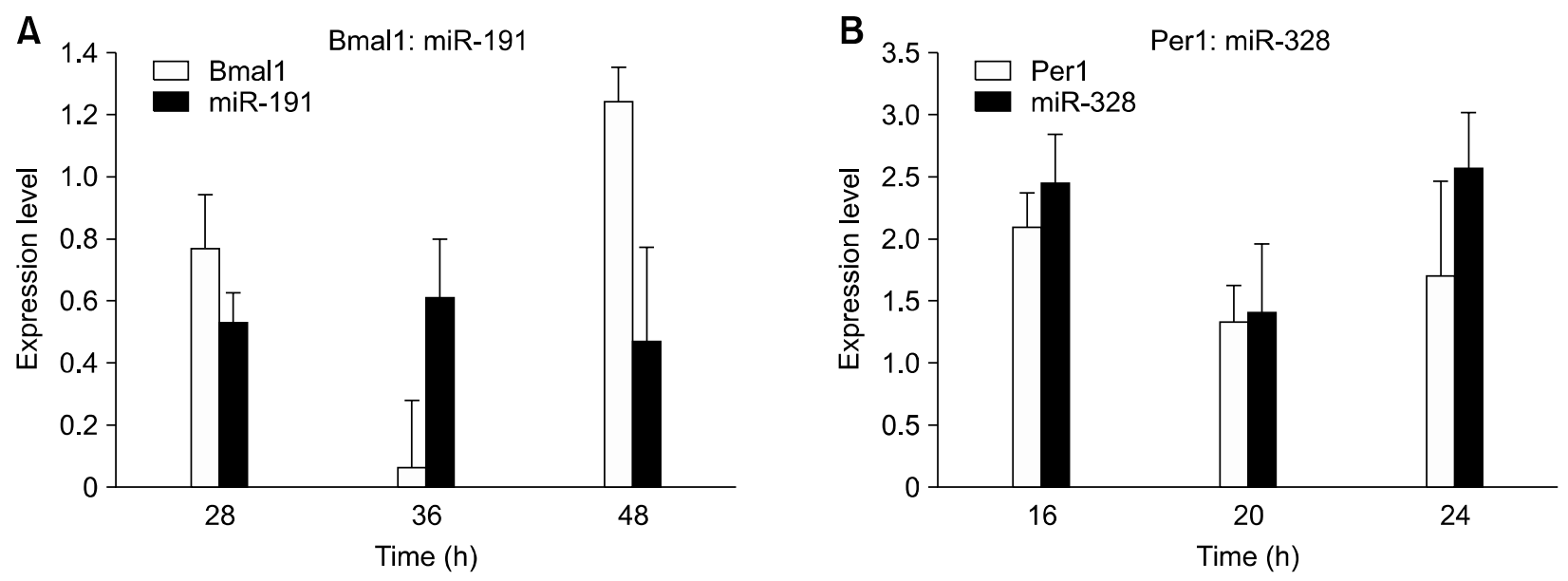

Figure 3. Verification of microarray results with RT-PCR. Mean values were determined from three independent experiments. Micro-RNA and mRNA levels were normalized to GAPDH levels and expressed as mean +/- SEM fold expression. (A) Expression patterns of miR-191 and Bmal1. (B) Expression patterns of miR-328 and Per1.

added.

Correlation analysis shows the inverse (Figure $2 \mathrm{~A}$ and $2 \mathrm{~B}$ ) and positive (Figure $2 \mathrm{C}-2 \mathrm{E}$ ) correlations of expression patterns between targeting miRNA with circadian initiator and suppressor pairs, respectively. This finding strongly suggests that miRNAs may control circadian rhythm by regulating circadian transcription factors, Clock:Bmal1, and the negative feedback regulators. For validation, we performed RT-PCR on miR-191-Bmal1 and miR328-Per1 pairs isolated from mouse liver, confirming robust circadian expression patterns (Figure 3 ).

\section{Genomic locations}

Genomic distribution of cyclic and non-cyclic miRNAs targeting circadian genes was investigated (Table 3) using the miRBase Sequences database (Griffiths-Jones, 2004; Griffiths-Jones et al., 2006). While the relative frequencies of cyclic and noncyclic ones among the miRNAs that do not target circadian genes did not show statistical difference ( $P$ value $=0.118$ by Fisher's exact test) across different regions of the genome, those targeting circadian genes showed significant difference $(P$ value $=0.013$ by Fisher's exact test). Further analysis revealed that intronic miRNAs targeting circadian genes showed significantly higher abundance of cyclic versus non-cyclic miRNAs $(17: 53=0.321$, $P$ value $=0.0007$ by Fisher's exact test) than all other regions $(P$ value $>0.1)$ including intergenic $(12: 136=0.088)$, exonic $(0: 6=0.0), 3$ 'UTR $(0: 10=$ $0.0)$, unknown $(1: 6=0.167)$ and the whole genome $(24: 181=0.132)$ regions $($ Table 3$)$.

\section{Discussion}

The present study showed the specific positive and negative correlation patterns of miRNA-mRNA target pairs in the circadian rhythm. Clock and Bmal1, which function as circadian transcription factors (Gekakis et al., 1998; Bunger et al., 2000), showed inversely correlated circadian expression patterns against their corresponding miRNAs, miR-181d and miR-191. In contrast, the components of negative feedback loop in the circadian rhythm, Per1 and Cry1, subject to Clock:Bmal1-mediated activation (Shearman et al., 2000), exhibited positively correlated circadian expression patterns to their

Table 3. Genomic distribution of cyclic and non-cyclic miRNAs targeting circadian genes.

\begin{tabular}{lcccccc}
\hline \multicolumn{1}{c}{ miRNAs } & Intron $^{*}$ & Inter-genic & Exon & 3'-UTR & Unknown & Whole genome \\
\hline miRNAs targeting circadian genes & & & & & & \\
miRNAs with cyclic expression pattern & 17 & 12 & 0 & 0 & 1 & 24 \\
miRNAs without cyclic expression pattern & 53 & 136 & 6 & 10 & 6 & 181 \\
ratio between cyclic and non-cyclic ones & 0.321 & 0.088 & 0.000 & 0.000 & 0.167 & 0.132 \\
\hline
\end{tabular}

Total count of probes can be different from the sum of miRNAs according to the genomic region because miRNAs may be located at multiple genomic regions. ${ }^{*} P<0.05$ after Bonferroni correction. 
corresponding miRNAs, miR-328 and miR-383.

Because miRNAs have been known to act as potent silencers of gene expression via translational repression or mRNA degradation as demonstrated in the circadian clock initiator, Clock:Bmal1 heterodimer, positive expression patterns between miRNAs and their targets as demonstrated in the circadian clock repressor, Per:Cry heterodimer, are unexpected. However, it has been shown that miRNAs may also function to induce gene expression (Place et al., 2008). Another possible explanation is that there can be a time lag between the inhibitory reaction and the resultant inhibition, resulting in positive correlation.

To characterize the targets of miRNAs with cyclic expression patterns, we used TarBase that houses a manually curated collection of experimentally tested miRNA targets, in view of considerable noise in miRNA target prediction. Experimental techniques used in TarBase are in vitro reporter gene assay and transgenic mice over-expressing a miRNA of interest. Verified targets of miRNAs with cyclic expression patterns are oncogenes like KRAS and $K I T$ and cell cycle regulatory protein like E2F1. Besides, $F B X W 1 B$ belonging to F-box protein family such as $F B X L 3$, which control the circadian clock by directing the degradation of cryptochrome proteins is included. It is suggested that miRNAs with circadian expression patterns may be involved in the cyclic activity.

We found that cyclic miRNAs targeting circadian genes show relatively high frequency in the intron region compared to other regions. The biological significance of the observations is unclear. One possible explanation, however, is that some genes could quickly and exactly regulate their expression patterns by their intronic miRNAs, circumventing the long-delayed translation process in rapidly shifting environments (Lin et al., 2006). Protein-coding genes produce not only gene transcripts for its own protein synthesis but also intronic miRNAs, which may suppress the expression of other genes. In other words, those gene expressions may result in gain-of-function of itself and loss-of-function of others, having complementarity to the mature intronic miRNA. We can speculate that intron-mediated gene regulation may be as much important as transcription factor-mediated regulation of gene expression.

We attempt to define criteria for a miRNA to be a potential regulator of the circadian rhythm: (i) one displays cyclic expression pattern, (ii) one targets circadian genes, and (iii) one shows inverse correlation of expression pattern with predicted target (s). Target genes of miRNAs that were determined to satisfy these requirements were clock initiators,
Clock and Bmal1 (Table 2, Figure 2 and 3). This finding indicates that miRNAs may specifically regulate circadian transcription factors among the several circadian rhythm-related genes. Thus, miRNAs may play a pivotal role in regulating circadian rhythm through circadian transcription factors.

Up to now, researchers have made many attempts to identify clock-associated miRNAs and their function in the circadian rhythm (Pegoraro and Tauber, 2008). Cheng et al. have revealed that miR-219 and miR-132 were shown to regulate the circadian clock in the mouse suprachiasmatic nucleus (Cheng et al., 2007). Another approach reported that circadian clocks regulate miRNA expression (dme-miR-263a and -263b) in Drosophila (Yang et al., 2008). Compared with their studies, one strength of the present study comes from the strategy of high-throughput co-expression analysis of mRNA and miRNA in the course of circadian rhythm. The circadian rhythm provides a strong constraint of cyclicity on both mRNA and miRNA expressions that filters out non-cyclic ones. Both RNAs are interconnected by the sequence-based target relationship that filters out non-interacting pairs. Cyclic expression of both RNAs can be further constrained by the statistical significance of correlation pattern analysis. These constraints enable an effective exploration of the plethora of complex networks.

Our study suggested that miRNAs including miR-181d and miR-191 are involved in the regulation of circadian rhythm by controlling the circadian transcription factor in mouse liver. Further study is required to identify the specific targets and networks of miRNAs in the circadian rhythm. Identifying specific miRNAs and their targets that are critically involved in circadian rhythm will provide a better understanding of circadian-clock regulation. This research suggests a previously unexplored layer of modulation to the circadian clock initiators through miRNAs.

\section{Methods}

\section{Samples}

We purchased 36 male C57BL/6J mice of 12 weeks of age. We entrained the mice to 12-h light and 12-h dark cycle (LD12:12) for four weeks. Liver sample were collected from three mice for each of the 12 time points with four-h interval for two complete circadian cycles. Hybridization was duplicated in miRNA and triplicated in mRNA expression profiling for each of the 12 pooled samples. Total RNA (including miRNA) from frozen liver tissues was isolated by using the mirVana RNA Isolation kit (Ambion, Austin, TX) according to the manufacturer's protocol. 


\section{MicroRNA microarray hybridization}

MiRNA isolated from the liver samples were labeled using the mirVana miRNA Array Labeling Kit (Ambion, Austin, TX) and then fluorescently coupled with Cy3 or Cy5 using the NHS-ester CyDye ${ }^{\mathrm{TM}}$ fluors (Amersham Bioscience, Pittsburgh, PA). Hybridization to the mirVana miRNA Bioarrays (Ambion, Austin, TX) was conducted in duplicate with the mirVana miRNA Bioarray Essentials Kit (Ambion, Austin, TX). The mirVana technology (Ambion, Austin, TX) profiles 640 human (hsa-mir, $n=328$ ), mouse (mmu-mir, $n$ $=114$ ) and rat (rno-mir, $n=46$ ), and Ambion proprietary human (ambi-mir, $n=152$ ) miRNA sequences. It can be considered that there are in fact 266 mouse miRNA probes that consist of the mmu $(n=114)$ probes and the 152 among 328 human miRNA probes having well conserved sequence homology with mouse counterparts. Following hybridization, the miRNA arrays were scanned using a GenePix 4000A array scanner (Axon Instruments, Union City, CA).

\section{MessengerRNA microarray hybridization}

$550 \mathrm{ng}$ of total RNA isolated from mouse liver was reverse-transcribed to cDNA using a T7 oligo (dT) primer. Second-strand cDNA was synthesized and labeled with biotin-NTP. Biotin labeled cRNA probes synthesized with the Illumina TotalPrep RNA Amplification kit (Ambion). 0.85 $\mu \mathrm{g}$ of labeled cRNA samples were hybridized in triplicate to each Sentrix Mouse-6 Expression BeadChip (Illumina, Inc., San Diego, CA). Detection of array signal was carried out following the BeadChip manual. Arrays were scanned with an Illumina Bead array Reader confocal scanner. mRNA expression data have been submitted to the Gene Expression Omnibus (GEO; http://www.ncbi.nlm.nih.gov/geo) with the series accession number GSE11516.

\section{RT-PCR}

We treated $20 \mu \mathrm{g}$ of total RNA with DNase (Ambion). cDNA was synthesized with an NCodeTM miRNA First Strand cDNA Synthesis RT-PCR kit (Invitrogen) from 200 ng of treated RNA. PCR primers were as follows: mmumiR-191, 5'-CAACGGAATCCCAAAAGCAGCTG; has-miR328, 5'-CTGGCCCTCTCTGCCCTTCC; Bmal1, 5'-AAGTGCAACAGGCCTTCAGT (sense strand) and 5'-GGTGGCCAGCTTTTCAAATA (antisense strand); Per-1, 5'-CTTCCCCCTGCTCCCACATC (sense strand) and 5'-AGTGGGAGGCCCTTCAGCAG (antisense strand). Quantitative PCR was done in triplicate on a 7000 Real Time PCR System (Applied Biosystems). For liver tissue sample, PCR was done in triplicate to minimize the variation. GAPDH was used as an endogenous control for gene expression.

\section{Statistical analysis}

The mRNA data set was normalized by "chip-wise" method using the rank invariant algorithm (Kuhn et al., 2004) and the miRNA data set was normalized by quantile normalization (Bolstad et al., 2003). We applied two statistical methods to find the genes showing circadian rhythm. We first applied the cosinor analysis method $(r>0.7$ and $P$ value $<0.05$ ). A least-square cosine wave was fitted to the data to test for the periodicity of $24 \mathrm{~h}$. Second, to find the genes that have periodic expression patterns that are not similar to cosine curves, we extracted the genes having significant positive correlation (Pearson correlation coefficient) between the first and the second day expression patterns $(r>0.7$ and $P$ value $<0.05)$. We extracted 85 miRNAs with diurnal expression pattern by combining the results of the two methods. Fisher's exact test was applied to examine the difference between cyclic and non-cyclic expression patterns across genomic regions.

\section{Acknowledgements}

This study was supported by a grant from the Ministry of Science and Technology, Republic of Korea (M1072907000107N290700110) and in part by a grant of the Korea Health 21 R\&D Project, Ministry of Health, Welfare and Family, Republic of Korea (0405-BC02-0604-0004).

\section{References}

Bartel DP. MicroRNAs: genomics, biogenesis, mechanism, and function. Cell 2004;116:281-97

Bolstad BM, Irizarry RA, Astrand M, Speed TP. A comparison of normalization methods for high density oligonucleotide array data based on variance and bias. Bioinformatics 2003;19:185-93

Bunger MK, Wilsbacher LD, Moran SM, Clendenin C, Radcliffe LA, Hogenesch JB, Simon MC, Takahashi JS, Bradfield CA. Mop3 is an essential component of the master circadian pacemaker in mammals. Cell 2000;103:1009-17

Cheng HY, Papp JW, Varlamova O, Dziema H, Russell B, Curfman JP, Nakazawa T, Shimizu K, Okamura H, Impey S, Obrietan K. microRNA modulation of circadian-clock period and entrainment. Neuron 2007;54:813-29

Gekakis N, Staknis D, Nguyen HB, Davis FC, Wilsbacher LD, King DP, Takahashi JS, Weitz CJ. Role of the CLOCK protein in the mammalian circadian mechanism. Science 1998;280: 1564-9

Griffiths-Jones S. The microRNA Registry. Nucleic Acids Res 2004;32:D109-11

Griffiths-Jones S, Grocock RJ, van Dongen S, Bateman A, Enright AJ. miRBase: microRNA sequences, targets and gene nomenclature. Nucleic Acids Res 2006;34:D140-4

Hornstein E, Mansfield JH, Yekta S, Hu JK, Harfe BD, McManus MT, Baskerville S, Bartel DP, Tabin CJ. The microRNA miR-196 acts upstream of Hoxb8 and Shh in limb development. Nature 2005;438:671-4

John B, Enright AJ, Aravin A, Tuschl T, Sander C, Marks DS. Human MicroRNA targets. PLoS Biol 2004;2:e363

Kiriakidou M, Nelson PT, Kouranov A, Fitziev P, Bouyioukos C, Mourelatos Z, Hatzigeorgiou A. A combined computational-experimental approach predicts human microRNA targets. Genes Dev 2004;18:1165-78

Krek A, Grun D, Poy MN, Wolf R, Rosenberg L, Epstein EJ, 
MacMenamin P, da Piedade I, Gunsalus KC, Stoffel M, Rajewsky N. Combinatorial microRNA target predictions. Nat Genet 2005;37:495-500

Kuhn K, Baker SC, Chudin E, Lieu MH, Oeser S, Bennett H, Rigault P, Barker D, McDaniel TK, Chee MS. A novel, highperformance random array platform for quantitative gene expression profiling. Genome Res 2004;14:2347-56

Lagos-Quintana M, Rauhut R, Lendeckel W, Tuschl T. Identification of novel genes coding for small expressed RNAs. Science 2001;294:853-8

Lau NC, Lim LP, Weinstein EG, Bartel DP. An abundant class of tiny RNAs with probable regulatory roles in Caenorhabditis elegans. Science 2001;294:858-62

Lee RC, Feinbaum RL, Ambros V. The C. elegans heterochronic gene lin- 4 encodes small RNAs with antisense complementarity to lin-14. Cell 1993;75:843-54

Lee RC, Ambros V. An extensive class of small RNAs in Caenorhabditis elegans. Science 2001;294:862-4

Lewis BP, Shih IH, Jones-Rhoades MW, Bartel DP, Burge CB. Prediction of mammalian microRNA targets. Cell 2003; 115:787-98

Lewis BP, Burge CB, Bartel DP. Conserved seed pairing, often flanked by adenosines, indicates that thousands of human genes are microRNA targets. Cell 2005;120:15-20

Lin SL, Miller JD, Ying SY. Intronic MicroRNA (miRNA). J Biomed Biotechnol 2006;2006:26818

Lowrey PL, Takahashi JS. Mammalian circadian biology: elucidating genome-wide levels of temporal organization. Annu Rev Genomics Hum Genet 2004;5:407-41

Pasquinelli AE, Reinhart BJ, Slack F, Martindale MQ, Kuroda MI, Maller B, Hayward DC, Ball EE, Degnan B, Muller P, Spring J, Srinivasan A, Fishman M, Finnerty J, Corbo J, Levine M, Leahy P, Davidson E, Ruvkun G. Conservation of the sequence and temporal expression of let-7 heterochronic regulatory RNA. Nature 2000;408:86-9

Pegoraro M, Tauber E. The role of microRNAs (miRNA) in circadian rhythmicity. J Genet 2008;87:505-11

Place RF, Li LC, Pookot D, Noonan EJ, Dahiya R. MicroRNA-373 induces expression of genes with complementary promoter sequences. Proc Natl Acad Sci U S A 2008;105: 1608-13

Reddy AB, Karp NA, Maywood ES, Sage EA, Deery M, O'Neill JS, Wong GK, Chesham J, Odell M, Lilley KS, Kyriacou CP, Hastings MH. Circadian orchestration of the hepatic proteome. Curr Biol 2006;16:1107-15

Reppert SM, Weaver DR. Molecular analysis of mammalian circadian rhythms. Annu Rev Physiol 2001;63:647-76

Sethupathy P, Corda B, Hatzigeorgiou AG. TarBase: A comprehensive database of experimentally supported animal microRNA targets. RNA 2006;12:192-7

Shearman LP, Jin X, Lee C, Reppert SM, Weaver DR. Targeted disruption of the mPer3 gene: subtle effects on circadian clock function. Mol Cell Biol 2000;20:6269-75

Siepka SM, Yoo SH, Park J, Song W, Kumar V, Hu Y, Lee C, Takahashi JS. Circadian mutant Overtime reveals F-box protein FBXL3 regulation of cryptochrome and period gene expression. Cell 2007;129:1011-23

Thomson JM, Parker J, Perou CM, Hammond SM. A custom microarray platform for analysis of microRNA gene expression. Nat Methods 2004;1:47-53

Weber MJ. New human and mouse microRNA genes found by homology search. FEBS J 2005;272:59-73

Wienholds E, Plasterk RH. MicroRNA function in animal development. FEBS Lett 2005;579:5911-22

Yang M, Lee JE, Padgett RW, Edery I. Circadian regulation of a limited set of conserved microRNAs in Drosophila. BMC Genomics 2008;9:83 